\title{
A review of trial and real-world data applying a realist approach to identify behavioural mechanisms supporting practitioners to taper opioids
}

\author{
Debi BHATTACHARYA ${ }^{1}$, Hattie Whiteside ${ }^{2}$, Emma Tang ${ }^{2}$, Kumud Kantilal $^{2}$, Yoon \\ Loke $^{2}$, Bethany Atkins ${ }^{2}$, and Caroline Hill ${ }^{2}$ \\ ${ }^{1}$ University of Leicester College of Life Sciences \\ ${ }^{2}$ University of East Anglia
}

December 2, 2021

\begin{abstract}
This realist enquiry applying behavioural theory aimed to identify behavioural mechanisms and contexts that facilitate prescribers tapering opioids. We identified relevant opioid tapering interventions and services from a 2018 international systematic review and a 2019 England-wide survey, respectively. Interventions and services were eligible if they provided information about contexts and/or behavioural mechanisms influencing opioid tapering success. A stakeholder group ( $\mathrm{n}=23)$ generated draft programme theories based around the 14 domains of the theoretical domains framework. We refined these using the trial and service data. From 71 articles and 21 survey responses, 56 and 16 respectively were included, representing primary care, hospital, specialist pain facilities and prison services. We identified six programme theories that included five behavioural mechanisms: prescribers' knowledge about how to taper; build prescribers' beliefs about capabilities to initiate tapering discussions and manage psychological consequences of tapering; perceived professional role in tapering; the environmental context enabling referral to specialists; and facilitating positive social influence by aligning patient: prescriber expectations of tapering. No interventions are addressing all six mechanisms supportive of tapering. Work is required to operationalise programme theories according to organisational structures and resources. An example operationalisation is combining tapering guidelines with information about local excess opioid problems and endorsing these with organisational branding. Prescribers being given the skills and confidence to initiate tapering discussions by training them in cognitive-based interventions and incorporating access to psychological and physical support in the patient pathway. Patients being provided with leaflets about the tapering process and informed about the patient pathway.
\end{abstract}

\section{Hosted file}

BJCP Manuscript 28.11.2021 clean.docx available at https://authorea.com/users/449264/ articles/547818-a-review-of-trial-and-real-world-data-applying-a-realist-approach-toidentify-behavioural-mechanisms-supporting-practitioners-to-taper-opioids 